\title{
Predicting adverse side effects of drugs
}

\author{
Liang-Chin Huang ${ }^{1}$, Xiaogang $\mathrm{Wu}^{1,2}$, Jake $\mathrm{Y} \mathrm{Chen}^{2,1^{*}}$ \\ From BIOCOMP 2010. The 2010 International Conference on Bioinformatics and Computational Biology \\ Las Vegas, NV, USA. 12-15 July 2010
}

\begin{abstract}
Background: Studies of toxicity and unintended side effects can lead to improved drug safety and efficacy. One promising form of study comes from molecular systems biology in the form of "systems pharmacology". Systems pharmacology combines data from clinical observation and molecular biology. This approach is new, however, and there are few examples of how it can practically predict adverse reactions (ADRs) from an experimental drug with acceptable accuracy.

Results: We have developed a new and practical computational framework to accurately predict ADRs of trial drugs. We combine clinical observation data with drug target data, protein-protein interaction (PPI) networks, and gene ontology $(\mathrm{GO})$ annotations. We use cardiotoxicity, one of the major causes for drug withdrawals, as a case study to demonstrate the power of the framework. Our results show that an in silico model built on this framework can achieve a satisfactory cardiotoxicity ADR prediction performance (median AUC $=0.771$, Accuracy $=0.675$, Sensitivity $=0.632$, and Specificity $=0.789$ ). Our results also demonstrate the significance of incorporating prior knowledge, including gene networks and gene annotations, to improve future ADR assessments.

Conclusions: Biomolecular network and gene annotation information can significantly improve the predictive accuracy of ADR of drugs under development. The use of PPI networks can increase prediction specificity and the use of GO annotations can increase prediction sensitivity. Using cardiotoxicity as an example, we are able to further identify cardiotoxicity-related proteins among drug target expanding PPI networks. The systems pharmacology approach that we developed in this study can be generally applicable to all future developmental drug ADR assessments and predictions.
\end{abstract}

\section{Background}

Systematic and quantitative studies of adverse side effects have become increasingly important due to rising concerns about the cytotoxicity of drugs in development [1]. According to the US Food and Drug Administration (FDA), up to $90 \%$ of all experimental drug compounds going through clinical trials fail to gain FDA approvals due to problems such in efficacy, formulation, pharmacokinetics (PK), toxicology, or clinical safety. In the past decade, concerns over drug toxicity have risen significantly (from $10 \%$ to $20 \%$ during the decade), while concerns over drug efficacy have remained unchanged (25-30\%) and concerns over drug PK have decreased significantly (from $40 \%$ to less than 10\%). It is time for

\footnotetext{
* Correspondence: j.chen@medeolinx.com

${ }^{2}$ MedeoLinx, LLC, Indianapolis, IN 46280, USA

Full list of author information is available at the end of the article
}

drug developers to design new and accurate models to assess unwanted side effects and drug actions before costly human clinical trials.

Recent research on the adverse side effects (ADR) [2] of drugs in cells has drawn attention to the inadequacy of the traditional "one drug, one target, and causal effect" model. Modern drugs are designed to regulate the functions of specific target proteins, or "drug targets". Efficacious drugs can break through human barriers of absorption, discretion, metabolism, and excretion to achieve desirable "on-target" effects. However, drugs may also bind to "off-target" proteins, potentially leading to unwanted side effects or ADRs, which range from mild drowsiness to deadly cardiotoxicity [3]. More appropriate models must be developed to take advantage of complex molecular responses of drugs in cells, by exploiting fully the relationships between
C Biomed Central 
chemical compounds, protein targets, and side effects observed at the physiological level [4]. The recent emergence of a systems approach to drug discovery has revitalized research in these areas of study [5,6]. By integrating the studies of human molecular networks, chemical compound similarity networks, and proteindrug association networks, systems biology researchers have spurred the development of systems pharmacology (also known as network pharmacology) [7][8]. By analyzing biological molecules and chemical entities in a variety of functional network contexts, drug developers can understand how drugs functions in a complex molecular system model [9], predict drug safety issues early $[10,11]$, identify ADR events early [12,13], and design diagnostic tests for tailoring drug treatments to individuals [14].

Although the importance between systems biology and ADR had been recognized before, there had been no published report about how to practically predict ADR from molecular annotation data until this study [15]. Mutsumi Fukuzaki et al. used cooperative pathways and gene expression profiles to predict ADRs [12]. Nonetheless, large-scale validations and performances were not mentioned. Based on the concept of ADR similarity analysis [4], Nir Atias et al. applied canonical correlation analysis and a network-based diffusion to predict ADRs [16], with prediction precision at merely less than 0.5 . Chemical structure-based approaches, also mentioned in Nir Atias' research, were used to predict off-targets [17] and ADRs [18]. While Andreas Bender et al. illustrated that this type of approach is quite sensitive in predicting $\mathrm{ADR}$, the positive predictive value is generally quite low, averaging under 0.5 [19]. Moreover, protein-protein interaction (PPI) networks were not employed for predicting adverse drug interactions until Lucas Brouwers et al. presented the contribution of PPI networks to drug side-effect similarities [15]; however, the prediction precision was too low $(=0.298)$ to be of practical significance.

In this work, we propose a computational systems pharmacology framework consisting of statistical modeling and machine learning to predict ADR of drugs. Our framework is based on comprehensive integration of systems biology data, including drugs, protein targets, molecular annotation, and reported side effects. The contribution of our work is the following: First, drugtarget interactions are expanded in global human PPI networks to build drug target expanding PPI networks. Second, drug targets are enriched by their gene ontology (GO) annotations to build drug target expanding GO networks. Third, ADR information for each drug is combined with drug target expanding PPI networks and drug target expanding GO networks. Fourth, statistics and machine learning are applied to build ADR classification/prediction models. Fifth, cross validation and feature selection are used to train prediction models. Without losing generality, we applied the framework to predict heart-related ADRs (i.e. drug cardiotoxicity), which are leading causes for clinical drug withdrawals [20]. The results of the cardiotoxicity prediction case study show that the performance of our approach (median AUC $=0.771$ ) is significantly better than all previously-reported studies. The positive contribution of PPI networks (including both topological and biological information) and the GO annotations (including only biological information) for drug cardiotoxicity prediction are also quantified for the first time.

\section{Results}

We report ADR prediction methods with cardiotoxicity as a case study. There are many ADRs related to cardiotoxicity, according to the index of the International Classification of Diseases 10th Revision (ICD-10) [21]. We merge all ADRs, each of which has an index ranging from I00 to I99 (classified as diseases of the circulatory system), into one group, $S_{H}$. The ADRs related to cardiotoxicity in SIDER and their ICD-10 indices are listed in Table 1. In the ADR vs. drug target (expanding network) facts (See the framework introduced in the Methods section), if any one of $D S_{n h}$ is 1 , where $D_{n}$ is drug $n$, and $S_{h}$ is in the group of heart-related ADR (see Table 1 ), then $D S_{n H}$ is set to 1 ; otherwise, $D S_{n H}$ is set to 0 . The mathematical details are described in the Methods.

We evaluated the performance of ADR predictions in multiple experiments by applying standard statistical performance-evaluation measures, i.e., AUC (area under ROC curve), ACC (accuracy), SEN (sensitivity), and SPE (specificity). For each evaluation experiment, we repeated 10 -fold cross validation three times and took median values to report prediction performances. To assess whether and how PPI networks (including both topological and biological information) or GO annotations (including only biological information) may contribute to a drug's cardiotoxicity-related ADR, we obtained results as described below.

\section{Use of biomolecular functional network data improves drug ADR predictions}

We examined drug ADR prediction performance by integrating different sets of confidence-ranked PPI data derived from the HAPPI database [22]. The database contains comprehensive human functional and physical protein interaction/association data, at different confidence levels, from "1 Star" (low confidence, mostly functional association data) to " 5 Star" (high confidence, mostly physical interaction data).

We can observe significant contributions of PPI networks to both prediction models, as shown in Figure 1 
Table 1 The ADRs related to cardiotoxicity in SIDER and their ICD-10 indices

\begin{tabular}{ll}
\hline ADRs in SIDER & ICD-10 Index \\
\hline Valvular Heart Disease & 108.8 \\
Rheumatic Carditis & 109.9 \\
Myocardial Infarction & 121 \\
Myocardial Ischemia & 125.6 \\
Heart Disease & $130-152$ \\
Constrictive Pericarditis & 131.1 \\
Pericardial Effusion & 131.3 \\
Cardiac Tamponade & 131.9 \\
Pericarditis & 132.8 \\
Endocarditis & 139.8 \\
Myocarditis & 140.8 \\
Cardiomyopathy & 142 \\
Second Degree Heart Block & 144.1 \\
Complete Heart Block & 144.2 \\
Heart Block & 145.5 \\
Cardiac Arrest & 146 \\
Sinus Tachycardia & 147 \\
Tachycardia & 147 \\
Junctional Tachycardia & 147.1 \\
Multifocal Atrial Tachycardia & 147.1 \\
Nodal Tachycardia & 147.1 \\
Supraventricular Tachycardia & 147.1 \\
Paroxysmal Ventricular Tachycardia & 147.2 \\
Ventricular Tachycardia & 147.2 \\
Heart Failure & 150 \\
Congestive Heart Failure & 150.0 \\
Right Heart Failure & 150.0 \\
Cardiomegaly & 151.7 \\
Cardiac Abnormality & 197.1 \\
\hline &
\end{tabular}

(a). When SVM (red solid line) is applied, the performance prediction goes up from AUC $=0.579$ (using "No Net", or not PPI network data) to AUC $=0.771$ (using "2 Stars UP" PPI network data). The use of PPI data bring up prediction performances significantly, i.e., Accuracy $=0.675$, Sensitivity $=0.632$, and Specificity $=$ 0.789. The increased AUC of the " 2 Stars UP" condition over the "No Net" condition is very significant, with $p$ value $=4.93 \mathrm{e}-35$ based on the $t$-test. If one further includes the lowest confidence level ("1-Star" PPI network data) into the drug target expanding network, the prediction performance decreases slightly due to noise in molecular networks. The performance curve of logistic regression (blue solid line) is comparable to, yet systematically lower than, that of SVM, moving up from AUC $=0.553$ (using "No Net") to AUC $=0.677$ (using "3 Stars UP" PPI network data). The performance of " 3 Stars UP" PPI network data is lower than that of "2 Stars UP" PPI network data, at Accuracy $=0.649$, Sensitivity $=0.564$ and Specificity $=0.789$. The increased

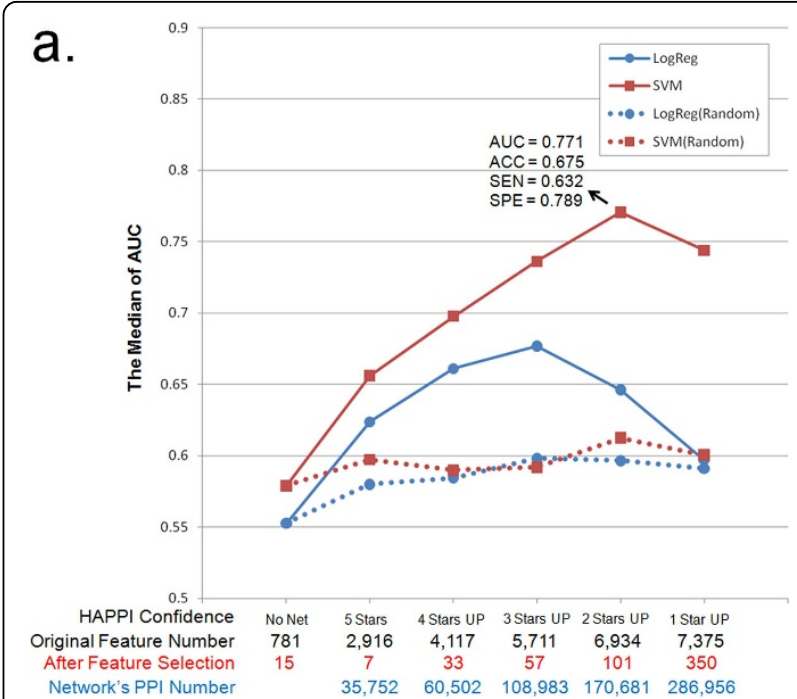

b.

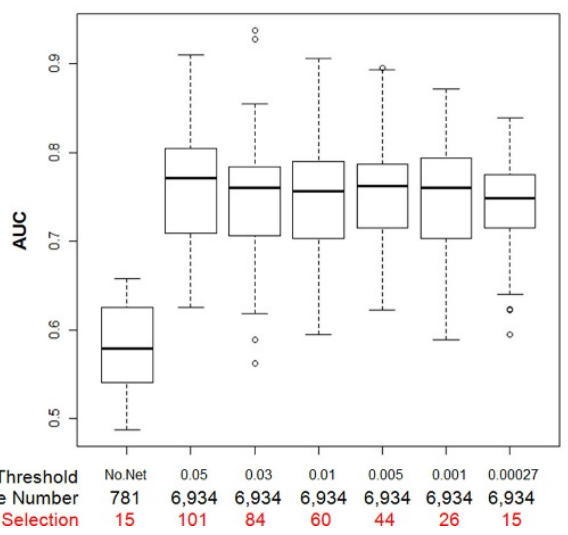

$\begin{array}{lccccccc}\text { Original Feature Number } & 781 & 6,934 & 6,934 & 6,934 & 6,934 & 6,934 & 6,934\end{array}$

Figure 1 The performances of SVM and logistic regression in the different confidence levels of PPIs. a) "No Net" means the prediction models used ADR vs. drug target facts; " 5 Stars" means we used PPIs in HAPPI under the confidence of 5 stars level; "4 Stars UP" means we used PPIs in HAPPI under the confidence of 4 to 5 stars level; and so forth. A red solid line represents the performance with SVM, while a blue solid line shows the performance with logistic regression. A red dotted line means we expanded the drug target network by replacing the PPI networks with random networks as control experiments or a base line of the performances with SVM, while a blue dotted line illustrates the effect of using logistic regression. AUC: area under ROC curve. ACC: accuracy. SEN: sensitivity. SPE: specificity. b) This box plot comparisons performances from using "2 Stars UP" PPI-expanding networks versus SVM under different $p$-value criteria in the feature selection.

AUC of the "3 Stars UP" condition over the "No Net" condition is also very significant, with $p$-value $=6.83 \mathrm{e}$ 18 based on the $t$-test. However, the decreased AUC performance between " 3 Stars UP" condition over the "2 Stars UP" condition is also noticeable, likely due to the functional nature (no longer biased towards physical PPI events) of biomolecular networks at the " 2 Stars" level reported by the HAPPI database. 
In order to control for the effects of using any types of (random) biomolecular networks and their possible contributions to ADR predictions, we also tested the model's performance with the use of randomized PPI networks which shared the same network topologies as actual PPI networks. Figure 1(a) shows that the performance curves using random networks slightly increased (with AUC > 0.55), when SVM (red dotted line) and logistic regression (blue dotted line) were applied. This result occurs because the original relationships between drugs and drug targets are still retained in the simulated random PPI networks. The additional gained prediction power, however, can only be explained by the embedded useful network information that our prediction model automatically learned from real biological network structures. These results strongly suggest that the contribution of PPI network data to drug ADR prediction is primarily due to useful functional information embedded in biomolecular functional association networks of drug targets and their related proteins, whereas network topology alone only plays a peripheral role.

We also studied whether the increase in our model's prediction performance may be due to the increase in the total number of features when PPI network data are introduced. For this purpose, we focus on the result obtained from the use of " 5 Stars" PPI network data, in which the number of features obtained by the prediction models becomes much smaller than that without using any network information. We noted that the AUC of this experimental result is better than that without using any network information ( $p$-value $=2.70 \mathrm{e}-8$ and $8.22 \mathrm{e}-9$ for $T$-test, when we used SVM and logistic regression, respectively). To further confirm the relationship between the number of features captured in the model and the model performance, we performed another experiment in which we gradually decreased feature number "2 Stars UP" PPI data in the SVM prediction model by lowering feature selection thresholds. Figure 1 (b) showed that there is no significant ( $p$-value $=0.469$ using ANOVA) decrease of prediction performances, when the number of features is filtered down. These observations further support our original finding that the contribution of PPI network for a drug's ADR prediction performance primarily comes from network data themselves.

\section{Integration of GO annotations also improves drug ADR predictions}

We also examined drug ADR prediction performance by integrating GO annotations available for each drug's protein targets. In two experiments (Figure 2), we directly incorporated into our prediction models GO annotation labels of drug target proteins. Since each

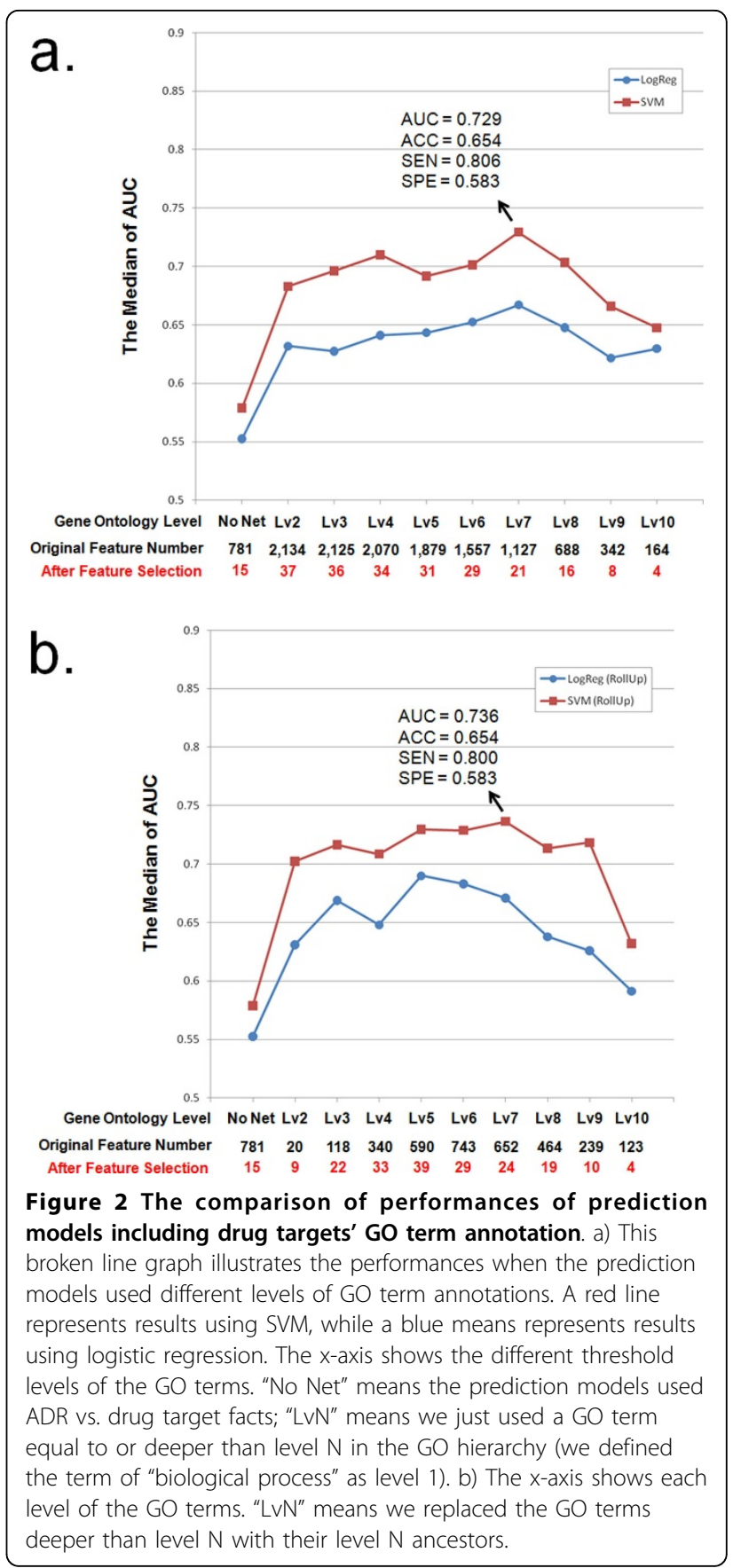

protein-coding gene may be annotated by many GO terms from different GO hierarchical levels, we carefully designed experiments to eliminate potential ADR prediction performance biases due to non-uniformity of GO term hierarchical levels. Therefore, we show in Figure 2 how GO terms aggregated to different GO hierarchical levels can contribute to prediction results based on different thresholds, the number of GO terms satisfying each threshold, and the number of GO terms selected into each model. Since GO hierarchical level = 
1 is not biologically meaningful and there is insufficient data for GO hierarchical levels from 11 to 15, results for these categories are not shown.

In the first experiment (Figure 2(a)), the GO terms equal to or deeper than specified threshold GO hierarchical levels are used to annotate drug targets for comparative drug ADR prediction performance analysis. Our results suggest that the prediction performances with the use of GO terms, regardless which predictive modeling method is used and which criteria is used for comparisons, are always better than those without the use of GO terms. In particular, when GO term level 7 (Lv7) is chosen, a best performance can be achieved with the use of SVM, in which we observed AUC = 0.729 and Sensitivity = 0.806; in comparison, "No Net" (without the use of GO term information) has AUC = 0.579 . The improvement in overall ADR prediction performance defined by AUC is significant ( $p$-value = $1.80 \mathrm{e}-18$, based on $t$-test).

In the second experiment (Figure 2(b)), the GO terms deeper than level $\mathrm{N}$ are replaced by their level $\mathrm{N}$ GO term ancestors to annotate drug targets for comparative drug ADR prediction performance analysis. We call this process a "Roll Up" and observed similar results as in the first experiment. In particular, when GO term Lv7 is chosen, a best performance can be achieved with the use of SVM, in which we observed AUC $=0.736$ and Sensitivity $=0.800$. The improvement in overall ADR prediction performance defined by AUC over the "No Net" experiment is also determined to be statistically significant ( $p$-value $=7.75 \mathrm{e}-17$, based on $t$-test).

Based on the above two experiments using GO terms, we can make the following conclusions. First, the use of GO annotations can help improve a drug's overall ADR prediction performance. Drug ADR prediction performances achieved with the best use of GO annotation $(\mathrm{AUC}=0.736)$ are almost comparable to those achieved with the best use of PPI networks $($ AUC $=0.771)$. Second, SVM models can help achieve better performance than logistic regression model can based on our case studies. Third, to achieve the best ADR prediction performance, it is best to choose SVM models and use GO biological process categorical terms at sufficiently detailed term levels (e.g., level 7) to annotate drug targets. Fourth, by evaluating detailed prediction performances achieved with PPI networks $(\mathrm{SEN}=0.632, \mathrm{SPE}=0.789)$ and GO annotations (SEN $=0.800, \mathrm{SPE}=0.583)$, we discovered that integration of biomolecular network data can increase the specificity (SPE) of ADR predictions, while the integration of GO annotation data can increase the sensitivity (SEN) of ADR predictions.

\section{A good ADR prediction model is concentrated not only on drug targets implicated with the ADR events, but also on many non-target proteins directly linked to ADR mechanisms}

We examined further the biological network contexts for 101 proteins selected automatically by the SVM prediction model as features. We expanded these "seed proteins" with "2 Stars UP" PPI interactions to build a PPI interaction network using the nearest neighborhood expansion method [23]. In Figure 3, we used node color and counts (in diamond shapes) to show how much evidence from PubMed might be identified in each protein.

Many selected proteins were found to have close relationships to cardiotoxicity. For example, ADRB1 (Adrenergic, beta-1-, receptor) mediates hormone epinephrine and neurotransmitter norepinephrine. The polymorphisms of ADRB1 have been shown to be involved in drug cardiotoxicity in heart failure [24]. Autoantibodies against the beta-1-adrenergic receptor have also been shown to have idiopathic dilated cardiomyopathy in some patients [25-27]. Therefore, it gives us great comfort that ADRB1 as a known drug target is also a part of the predictor.

We also observed that the drug target expanding network can bring forth additional cardiotoxicity-related non-target proteins, e.g., ERBB4 and CYP2D6. ERBB4, a v-erb-a erythroblastic leukemia viral oncogene homolog 4 , is a member of the type I receptor tyrosine kinase subfamily and encodes a receptor for NDF/heregulin. Targeted deletion and inhibition of ERBB4 signaling may lead to congestive heart failure resulting from cardiovascular defects $[28,29]$. CYP2D6 encodes a subunit of the cytochrome P450 superfamily of enzymes. The gene is specifically expressed in the right ventricle and its genetic polymorphism is known to be associated with cardiotoxicity, including a patient's poor anti-arrhythmic activity, severe cardiovascular, or dilated cardiomyopathy $[30,31]$.

Using all GO annotations from GO term level 7 "Rollups" to build predictors with the SVM model, we developed a final list of $24 \mathrm{GO}$ terms selected into the best cardiotoxicity prediction model (See Figure 2(b)). In Table 2, we list the 24 GO terms and their IDs. Interestingly, many of these terms appear to be related to heart disease or cardiotoxicity. There are many known literature reports linking these terms to cardiotoxicity. For example, Avkiran et al. [32] described the MAPKKK signaling cascades in heart failure; Yatani et al. [33] showed G proteins' roles in heart rate regulation; Plunkett et al. [34] examined the role of dopamine receptor on the cardiovascular system; the GO terms of GO:0008016 and GO:0045823 are defined as the terms related to regulation of heart contraction; GO:0051924 


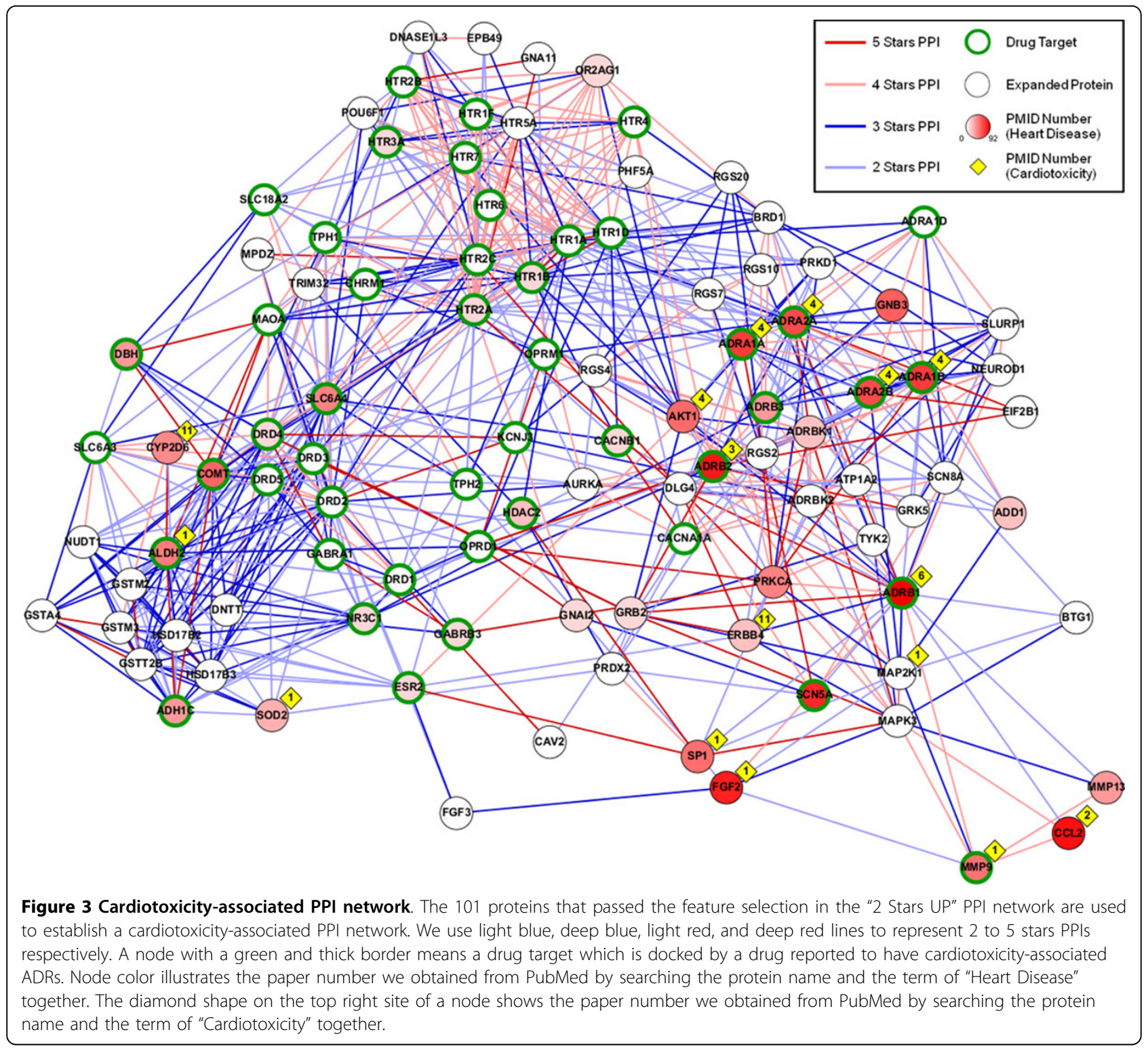

is involved in the regulation of calcium ion transport, a critical process in cardiovascular functions; Gosalvez et al. [35] showed evidence of the link between potassium transport and cardiotoxicity; and so on. Apparently, the prediction model works by integrating biologically significant drug targets within known cardiovascular side effects and the related non-target protein functional partners implied in cardiovascular functions or cardiotoxicity.

\section{Discussion}

In this work, we not only built effective ADR prediction models, but also showed that the use of biomolecular networks or gene annotations may independently improve ADR prediction performances. Integrating gene network and gene annotation allows the use of deeper level of biological knowledge [36] to increase a model's prediction performance. In future work, it would be preferable to study functional relationships between proteins that are not directly associated. Additional experimentally-based genotype-phenotype information, e.g., those derived from genome-wide association studies, may also be useful, as several recent studies of genetic polymorphisms of cardiotoxicity-inducing enzymes have already showed $[37,38]$.

We developed a general conceptual framework and demonstrated how to build practical ADR prediction models, using cardiotoxicity as a case study. For other drug ADR predictions, hepatotoxicity and nephrotoxicity are also critical issues to consider in drug development. 
Table 2 GO terms that passed the feature selection in the replaced level 7 terms

\begin{tabular}{ll}
\hline ID & GO Term \\
\hline GO:0000165 & MAPKKK Cascade \\
GO:0002031 & G-Protein Coupled Receptor Internalization \\
GO:0007194 & Negative Regulation of Adenylate Cyclase Activity \\
GO:0007210 & Serotonin Receptor Signaling Pathway \\
GO:0007212 & Dopamine Receptor Signaling Pathway \\
GO:0007612 & Learning \\
GO:0007613 & Memory \\
GO:0008016 & Regulation of Heart Contraction \\
GO:0009123 & Nucleoside Monophosphate Metabolic Process \\
GO:0019935 & Cyclic-Nucleotide-Mediated Signaling \\
GO:0043268 & Positive Regulation of Potassium lon Transport \\
GO:0043278 & Response to Morphine \\
GO:0043408 & Regulation of MAPKKK Cascade \\
GO:0045762 & Positive Regulation of Adenylate Cyclase Activity \\
GO:0045823 & Positive Regulation of Heart Contraction \\
GO:0045859 & Regulation of Protein Kinase Activity \\
GO:0045893 & Positive Regulation of Transcription, DNA-Dependent \\
GO:0046488 & Phosphatidylinositol Metabolic Process \\
GO:0051924 & Regulation of Calcium Ion Transport \\
GO:0051932 & Synaptic Transmission, Gabaergic \\
GO:0051937 & Catecholamine Transport \\
GO:0051969 & Regulation of Transmission of Nerve Impulse \\
GO:0072511 & Divalent Inorganic Cation Transport \\
GO:2000147 & Positive Regulation of Cell Motility \\
\hline &
\end{tabular}

Decision trees were developed and trained by others to predict hepatotoxicity and nephrotoxicity [39], relying on a drug's chemical properties to predict ADRs. Therefore, we plan to soon assess hepatotoxicity and nephrotoxicity in a future extension of this work and compare our performances with those of other approaches.

\section{Conclusion}

In this study, we presented a systems pharmacology framework for predicting drugs' ADR, using cardiotoxicity as an example. Our method is based on SVM and logistic regressions, by integrating ADR information, drug-target data, PPI networks, and GO term annotations. Our results indicated that integrating functional biomolecular association networks or detailed GO annotation could significantly improve a drug's ADR prediction. Particularly, comprehensive functional biomolecular association networks are shown to be useful for increasing specificity, while detailed gene annotation information are shown to be useful for increasing sensitivity. Moreover, proteins used to automatically build the prediction models are shown to further reveal related biological functions for cardiovascular health and cardiotoxicity. Overall, our study described a novel way of predicting ADRs, with comprehensive incorporation of additional prior knowledge ADR assessments.

\section{Methods}

A framework for ADR prediction is shown in Figure 4, which includes drug and network information retrieval, feature selection, cross validation, sample balancing, prediction models, and performance assessment. There are two types of data flows in the flowchart: 1) Black arrows indicate data flows for ADR vs. drug target facts. 2) Green arrows indicate data flows for ADR vs. drug

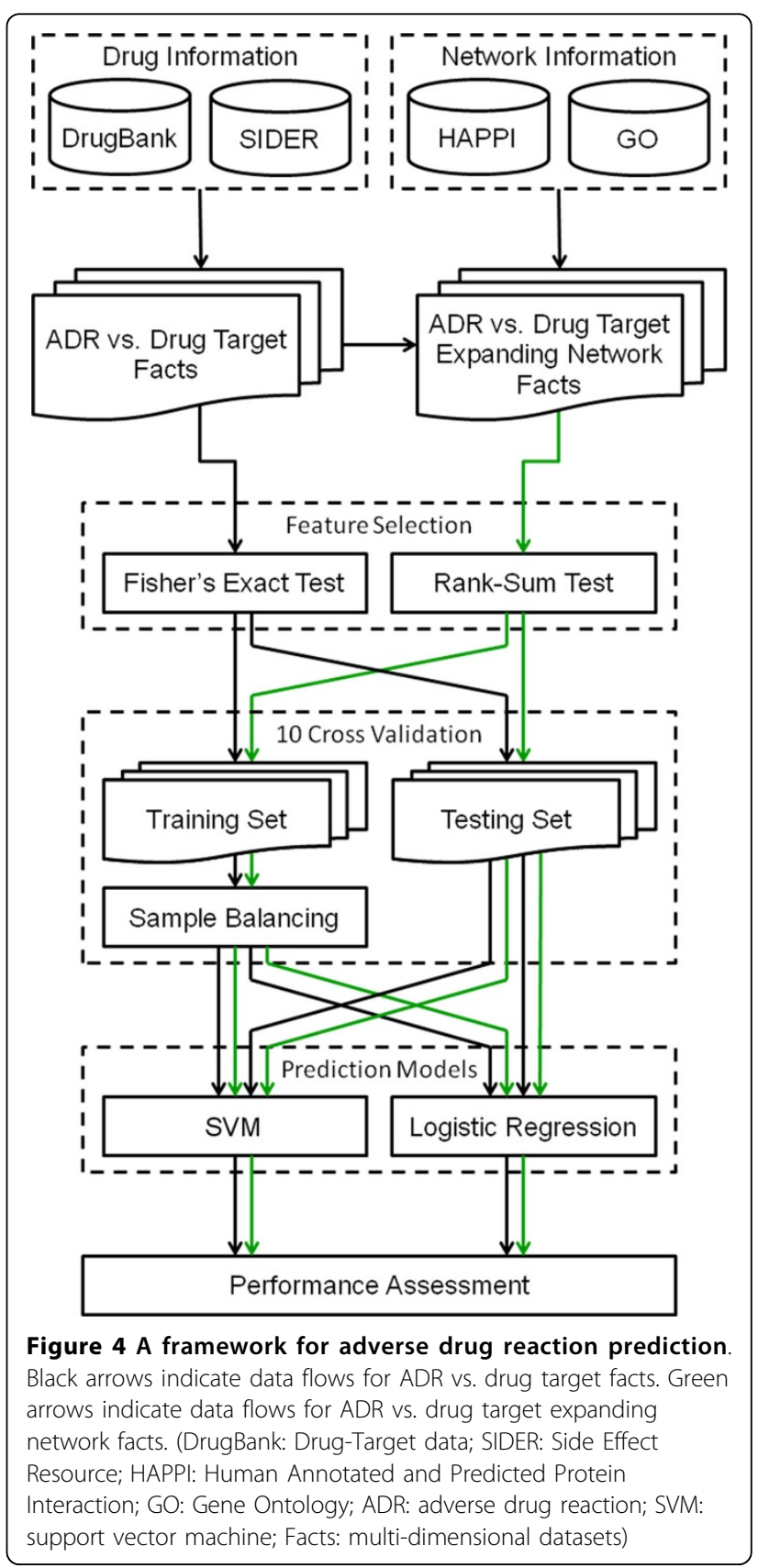


target expanding network facts, which are generated by integrating ADR vs. drug target facts and network information.

\section{Drug and network information retrieval}

We used three major public databases in our study.

First, the DrugBank database is exploited as a bioinformatics and cheminformatics resource, which contains drug and drug target information [40]. Up to May 2011, there were 5,461 drugs and 3,880 proteins, which formed 13,457 unique drug-target pairs in DrugBank, and they were extracted as main drug target information in this study.

Second, the Side Effect Resource (SIDER) database is also involved. This database aggregates FDA drug labels and disperses public information on ADRs [41]. There were 877 drugs, 1,447 kinds of ADR, and 61,824 relationships among drugs and ADRs obtained from COSTART and Euphoria-related ADRs in SIDER. There are 578 drugs overlapped between DrugBank and SIDER.

Third, the Human Annotated and Predicted Protein Interactions (HAPPI) database [22] is used as a global human PPI resource. HAPPI integrates HPRD, BIND, MINT, STRING, and OPHID. Most importantly, HAPPI provides a confidence star quality rating from 1 to 5 for each interaction based on the initial data sources, data generation methods, and number of literature references for the interaction. Excluding self PPIs, there are 116,275 PPIs, 61,698 PPIs, 48,481 PPIs, 24,750 PPIs, and 35,752 PPIs involved in the data set from 1 star to 5 stars, respectively. This data can be used to expand the network of drug targets.

Finally, the Gene Ontology (GO) project provides hierarchical terms, including biological processes, cellular components, and molecular functions, to describe the characteristics and annotations of gene product [42]. In this study, we only use biological processes, from a general term "biological process" in level 1 to specific terms in level 15, to expand the features in the prediction models from drug targets to the GO terms in order to investigate the biological meanings between drug targets and ADRs. There are 3,715 biological process terms utilized for annotating the drug targets in this study.

\section{ADR vs. drug target (expanding network) facts}

By combining the drug target information in DrugBank with the ADR information in SIDER, we obtained the ADR vs. drug target facts. The facts follow the format shown in Figure 5(a). If drug $n$ has a side effect $j$, the value in cell $D S_{n j}(n=1 \ldots N$, and $j=1 \ldots)$ at the intersection of column $S_{j}$ and row $D_{n}$ is 1 or "TRUE"; otherwise, it is 0 or "FALSE". So does the value in cell $D T_{n k}(n=$ $1 \ldots N$, and $k=1 \ldots K)$ at the intersection of column $T_{k}$ and row $D_{n}$ if drug $n$ docks to drug target $k$. The binary data

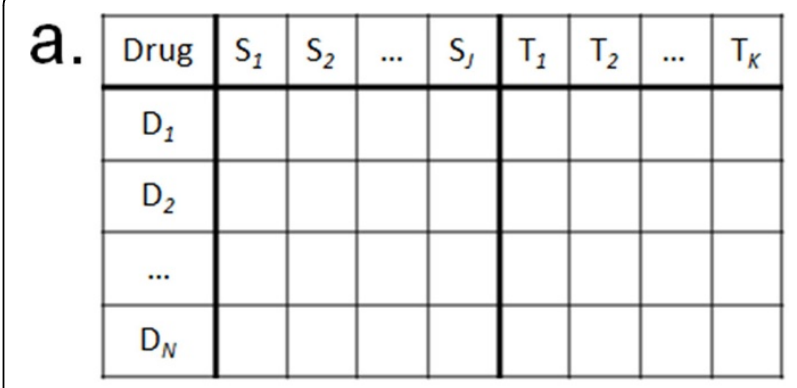

b. Drug Target Expanding Network

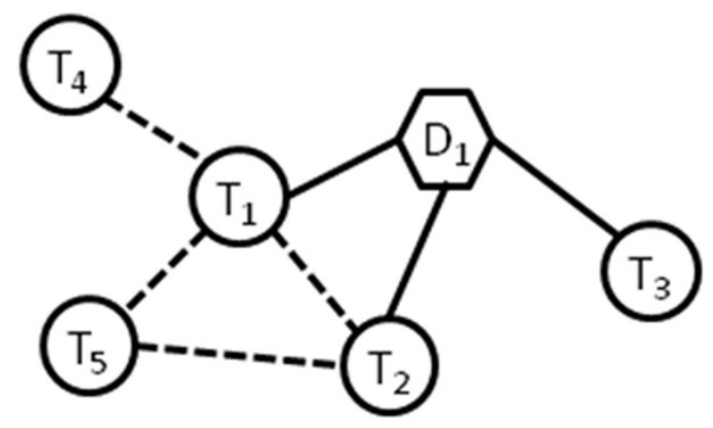

C.

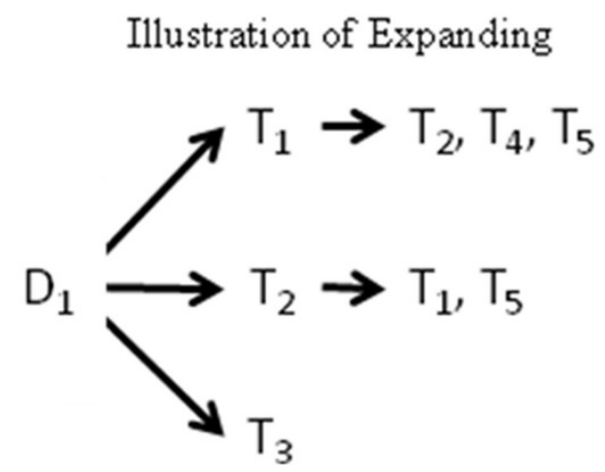

Figure 5 Format of ADR vs. drug target facts and an example of drug target expanding network. a) This figure shows the format of ADR vs. drug target facts. $D_{n}, S_{i}$, and $T_{j}$ mean drug $n$, ADR $i$, and target $j$ respectively. $b$ ) This figure is an example of a drug target expanding network. The dotted lines mean PPIs. c) This part illustrates the process of the drug target expanding one level in PPI network. $T_{1}, T_{2}$, and $T_{5}$ are present twice in all branches of this expanding "tree", thus the values in cells $D T_{11}, D T_{12}$, and $D T_{15}$ in ADR vs. drug target facts are all 2; the rest, including $D T_{13}$ and $D T_{141}$ are both 1.

$D S_{n j}$ and $D T_{n k}$, representing the ADR vs. drug target facts, can be then used for prediction model training and testing: each ADR $S_{j}$ is prediction output (response variable) and targets from $T_{1}$ to $T_{K}$ are features (dependent variables).

When the drug targets expand one level in a PPI network or are annotated by using the GO terms, the value in cell $D T_{n k}$ will be integer instead of binary, because the association between drug $n$ and drug target $k$ could 
be repeatedly present in the drug target expanding network. Figure 5b shows an example of a drug target expanding network, and Figure $5 \mathrm{c}$ shows the drug target expanding process and the repeated presences of $T_{1}, T_{2}$, and $T_{5}$. The repeat number here can be regarded as the weight of the relationship between drug and target under network level; moreover, this weight can be also used to study the contribution of each target to ADRs.

\section{Feature selection}

Since thousands of features (drug targets) are required to build prediction models, this process is exhaustive and memory consuming. Moreover, some statistics tools, such as R, have memory limitations [43]. Hence, it is necessarily to filter out the features that would make little contribution to the response variable. If the data type of cell $D T_{n k}$ is binary, Fisher's exact test will be used; otherwise, Wilcoxon rank-sum test will be used. In both methods, features will be selected if their $p$ values are smaller than 0.05 .

\section{Sample balancing}

The sample sizes of output classes are usually bias and imbalance, especially in medical data [44]. Consequently, the accuracy of the prediction result would be overestimated. In order to prevent this problem from happening, sample balancing method is also applied in this study. First, we randomly separate the major class into many parts. Each part contains a sample size close to that of the minor class. Second, we combine every part of the major class with the minor class as training sets. The input data is separated into ten parts in the process of 10 -fold cross validation: nine parts are taken to do the sample balancing and the remaining one is used to validate the prediction models. The training sets are balanced, while the validation set is still imbalanced in the sample sizes of classes, so the performance will be more reliable.

\section{Prediction models}

For comparisons, the prediction models in this study include two independent procedures: 1) machine learning - support vector machines (SVM), and 2) statistical modeling - logistic regression. A SVM package of R, called "e1071" [45] is used in this study. For kernel functions, we choose a nonlinear one: Gaussian radial basis function, which is also the optimized kernel function. This SVM package provides fitted probabilities numerically from 0 to 1 , and so does the logistic regression package used in this study, named as "generalized linear models" [46].

\section{Acknowledgements}

We thank Frank Smith, Heather McFall, Tulip Nandu, and Bryan Richards for help with preliminary proofreading. We especially thank Lun-Ching Chang and I-Chen Chen for statistical consultation. We also thank our anonymous reviewers for comments that enabled us to resolve deficiencies for this manuscript.

This article has been published as part of BMC Genomics Volume 12 Supplement 5, 2011: BIOCOMP 2010 - The 2010 International Conference on Bioinformatics \& Computational Biology: Genomics. The full contents of the supplement are available online at http://www.biomedcentral.com/1471$2164 / 12$ ? issue $=$ S5.

\section{Author details}

${ }^{1}$ School of Informatics, Indiana University, Indianapolis, IN 46202, USA. ${ }^{2}$ MedeoLinx, LLC, Indianapolis, IN 46280, USA

\section{Authors' contributions}

JYC conceived this work, guided the research team by providing ideas and feedback along the way, and revised the manuscript. LH integrated the information in public datasets, developed the analysis workflow, chose classifiers, and assessed the performance of the analysis. XW introduced SIDER to this study, specified mathematical descriptions, refined results, and helped improve the readability of the manuscript. All authors proofread and approved the final manuscript.

\section{Competing interests}

JYC and XW are co-founders of Medeolinx, LLC. This academic and private sector collaboration leverages Medeolinx's intellectual property, technologies, and equipment. However, this work does not promote any Medeolinxrelated products or services.

Published: 23 December 2011

\section{References}

1. Kola I, Landis J: Can the pharmaceutical industry reduce attrition rates? Nature Reviews Drug Discovery 2004, 3(8):711-716.

2. Kramer T: Side effects and therapeutic effects. MedGenMed 2003, 5(1):28.

3. Demetri GD: Structural reengineering of imatinib to decrease cardiac risk in cancer therapy. J Clin Invest 2007, 117(12):3650-3653.

4. Campillos M, Kuhn M, Gavin AC, Jensen LJ, Bork P: Drug target identification using side-effect similarity. Science 2008 321(5886):263-266.

5. Fishman MC, Porter JA: Pharmaceuticals: a new grammar for drug discovery. Nature 2005, 437(7058):491-493.

6. Werner E: Systems biology: the new darling of drug discovery? Drug Discov Today 2002, 7(18):947-949.

7. Hopkins AL: Network pharmacology: the next paradigm in drug discovery. Nature chemical biology 2008, 4(11):682-690.

8. Berger SI, lyengar R: Network analyses in systems pharmacology. Bioinformatics 2009, 25(19):2466.

9. Li J, Zhu X, Chen JY: Building disease-specific drug-protein connectivity maps from molecular interaction networks and PubMed abstracts. PLoS Comput Biol 2009, 5(7):e1000450.

10. Butcher EC, Berg EL, Kunkel EJ: Systems biology in drug discovery. Nat Biotechnol 2004, 22(10):1253-1259.

11. Ekins S, Nikolsky Y, Nikolskaya T: Techniques: application of systems biology to absorption, distribution, metabolism, excretion and toxicity. Trends Pharmacol Sci 2005, 26(4):202-209.

12. Mutsumi Fukuzaki MS, Hisashi Kashima, Jun Sese: Side Effect Prediction Using Cooperative Pathways. 2009 IEEE International Conference on Bioinformatics and Biomedicine 2009, 142-147.

13. Pouliot $Y$, Chiang AP, Butte AJ: Predicting adverse drug reactions using publicly available PubChem BioAssay data. Clin Pharmacol Ther 2011, 90(1):90-99.

14. Barabási AL, Gulbahce N, Loscalzo J: Network medicine: a network-based approach to human disease. Nature Reviews Genetics 2011, 12(1):56-68.

15. Brouwers $L$, Iskar $M$, Zeller $G$, van Noort V, Bork P: Network neighbors of drug targets contribute to drug side-effect similarity. PLoS One 2011, 6(7): e22187.

16. Atias N, Sharan R: An algorithmic framework for predicting side effects of drugs. J Comput Biol 2010, 18(3):207-218.

17. Keiser MJ, Setola V, Irwin JJ, Laggner C, Abbas Al, Hufeisen SJ, Jensen NH, Kuijer MB, Matos RC, Tran TB, et al: Predicting new molecular targets for known drugs. Nature 2009, 462(7270):175-181. 
18. Scheiber J, Jenkins JL, Sukuru SC, Bender A, Mikhailov D, Milik M, Azzaoui K, Whitebread S, Hamon J, Urban L, et al: Mapping adverse drug reactions in chemical space. J Med Chem 2009, 52(9):3103-3107.

19. Bender A, Scheiber J, Glick M, Davies JW, Azzaoui K, Hamon J, Urban L, Whitebread S, Jenkins JL: Analysis of pharmacology data and the prediction of adverse drug reactions and off-target effects from chemical structure. ChemMedChem 2007, 2(6):861-873.

20. Wilke RA, Lin DW, Roden DM, Watkins PB, Flockhart D, Zineh I, Giacomini KM, Krauss RM: Identifying genetic risk factors for serious adverse drug reactions: current progress and challenges. Nat Rev Drug Discov 2007, 6(11):904-916.

21. Geneva: The ICD-10 classification of mental and behavioural disorders: clinical descriptions and diagnostic guidelines. World Health Organization 1992.

22. Chen JY, Mamidipalli S, Huan T: HAPPI: an online database of comprehensive human annotated and predicted protein interactions. BMC Genomics 2009, 10(Suppl 1):S16.

23. Chen JY, Shen C, Sivachenko AY: Mining Alzheimer disease relevant proteins from integrated protein interactome data. Pac Symp Biocomput 2006, 367-378.

24. Ranade K, Jorgenson E, Sheu WH, Pei D, Hsiung CA, Chiang FT, Chen YD, Pratt $R$, Olshen RA, Curb $D$, et al: A polymorphism in the beta1 adrenergic receptor is associated with resting heart rate. Am J Hum Genet 2002, 70(4):935-942.

25. Magnusson Y, Marullo S, Hoyer S, Waagstein F, Andersson B, Vahlne A Guillet JG, Strosberg AD, Hjalmarson A, Hoebeke J: Mapping of a functional autoimmune epitope on the beta 1 -adrenergic receptor in patients with idiopathic dilated cardiomyopathy. J Clin Invest 1990, 86(5):1658-1663.

26. Bernstein D, Fajardo G, Zhao M, Urashima T, Powers J, Berry G, Kobilka BK: Differential cardioprotective/cardiotoxic effects mediated by betaadrenergic receptor subtypes. Am J Physiol Heart Circ Physiol 2005, 289(6): H2441-2449.

27. Christ T, Wettwer E, Dobrev D, Adolph E, Knaut M, Wallukat G, Ravens U: Autoantibodies against the beta1 adrenoceptor from patients with dilated cardiomyopathy prolong action potential duration and enhance contractility in isolated cardiomyocytes. J Mol Cell Cardiol 2001, 33(8):1515-1525

28. Fuller SJ, Sivarajah K, Sugden PH: ErbB receptors, their ligands, and the consequences of their activation and inhibition in the myocardium. $J$ Mol Cell Cardiol 2008, 44(5):831-854.

29. Horie $T$, Ono K, Nishi H, Nagao K, Kinoshita M, Watanabe S, Kuwabara $Y$, Nakashima Y, Takanabe-Mori R, Nishi E, et al: Acute doxorubicin cardiotoxicity is associated with miR-146a-induced inhibition of the neuregulin-ErbB pathway. Cardiovasc Res 2010, 87(4):656-664.

30. Thum T, Borlak J: Gene expression in distinct regions of the heart. Lancet 2000, 355(9208):979-983.

31. Ovaska H, Ludman A, Spencer EP, Wood DM, Jones AL, Dargan PI: Propafenone poisoning-a case report with plasma propafenone concentrations. J Med Toxicol 2010, 6(1):37-40.

32. Avkiran M, Marber MS: Feeling the stress: MAPKKK-MAPKK-MAPK signaling cascades in heart failure. J Mol Cell Cardio/ 2010, 48(2):283-285.

33. Yatani A, Okabe K, Codina J, Birnbaumer L, Brown AM: Heart rate regulation by $\mathrm{G}$ proteins acting on the cardiac pacemaker channel. Science 1990, 249(4973): 1163-1166.

34. Plunkett LM, Tackett RL: Central dopamine receptors and their role in digoxin-induced cardiotoxicity in the dog. J Pharm Pharmacol 1987, 39(1):29-34.

35. Gosalvez M, van Rossum GD, Blanco MF: Inhibition of sodium-potassiumactivated adenosine $5^{\prime}$-triphosphatase and ion transport by adriamycin Cancer Res 1979, 39(1):257-261.

36. Yunkyu Choi SK, Gwan-Su Yi, Jinah Park: Protein-Protein Interaction Network and Gene Ontology. In Future Application and Middleware Technology on e-Science Ok-Hwan Byeon JHK, Thom Dunning, Kum Won Cho, Aurore Savoy-Navarro 2010, 159-169.

37. Cornelis MC, El-Sohemy A, Kabagambe EK, Campos H: Coffee, CYP1A2 genotype, and risk of myocardial infarction. JAMA 2006, 295(10):1135-1141

38. Huang RS, Duan S, Kistner EO, Bleibel WK, Delaney SM, Fackenthal DL, Das S, Dolan ME: Genetic variants contributing to daunorubicin-induced cytotoxicity. Cancer Res 2008, 68(9):3161-3168
39. Hammann F, Gutmann H, Vogt N, Helma C, Drewe J: Prediction of adverse drug reactions using decision tree modeling. Clin Pharmacol Ther 2010, 88(1):52-59.

40. Knox C, Law V, Jewison T, Liu P, Ly S, Frolkis A, Pon A, Banco K, Mak C, Neveu V, et al: DrugBank 3.0: a comprehensive resource for 'omics' research on drugs. Nucleic Acids Res 2011, , 39 Database: D1035-1041.

41. Kuhn M, Campillos M, Letunic I, Jensen LI, Bork P: A side effect resource to capture phenotypic effects of drugs. Mol Syst Biol 2010, 6:343.

42. Ashburner M, Ball CA, Blake JA, Botstein D, Butler H, Cherry JM, Davis AP, Dolinski K, Dwight SS, Eppig JT, et al: Gene ontology: tool for the unification of biology. The Gene Ontology Consortium. Nat Genet 2000, 25(1):25-29.

43. Hornik K: The R FAQ. 2011

44. Oommen T: Sampling Bias and Class Imbalance in Maximum-likelihood Logistic Regression. Math Geosci 2011, 43:99-120.

45. Meyer : Support Vector Machines: The interface to libsvm in Package e1071. 2004.

46. Geyer CJ: Generalized Linear Models in R. 2003.

doi:10.1186/1471-2164-12-S5-S11

Cite this article as: Huang et al: Predicting adverse side effects of drugs. BMC Genomics 2011 12(Suppl 5):S11.

\section{Submit your next manuscript to BioMed Central and take full advantage of:}

- Convenient online submission

- Thorough peer review

- No space constraints or color figure charges

- Immediate publication on acceptance

- Inclusion in PubMed, CAS, Scopus and Google Scholar

- Research which is freely available for redistribution
C Biomed Central 